\title{
Triple bottom line: sustainability in amblyopia care
}

Eye (2016) 30, 1029-1030; doi:10.1038/eye.2016.116; published online 27 May 2016

Sustainability of healthcare is one of the guiding principles of Vision 2020 on the path to eliminating avoidable blindness ${ }^{1}$ and is actively promoted by the Royal College of Ophthalmologists. Sustainability involves balancing economic, environmental, and social outcomes and demands; this is the 'triple aim divided by the triple bottom line'. High-value healthcare should incur minimal economic and environmental impact. Previous work has used this framework to evaluate cataract surgery. 2,3

Attending hospital eye services (HES) can be difficult for families who often have several children to look after. In the amblyopia treatment pathway, many appointments for amblyopia treatment can safely be provided by orthoptist-led clinics. In our setting, protocols allow our HES and the orthoptist-led community clinics (CCs) offered by the local community healthcare provider to work seamlessly, so that most amblyopia appointments can take place in CCs. In addition to our regular clinical audits, we aimed to measure the impact of service provision on the 'triple bottom line'.

We compared the distance families travel to attend the HES $(n=92)$ and three CCs $(n=71)$, and the time spent in the clinic (appointment or arrival time to departure time, using the shorter of the two), during one week in November 2015 and one week in March/April 2016.

The median distance families travelled to the HES clinic was 6.4 miles (interquartile range IQR 2.5 -12.4), and to a CC 3.7 miles (IQR 1.1 - 5.1) (Figure 1, Table 1). The median time in clinic was $82 \mathrm{~min}$ for HES (IQR 55 to 107) and $20 \mathrm{~min}$

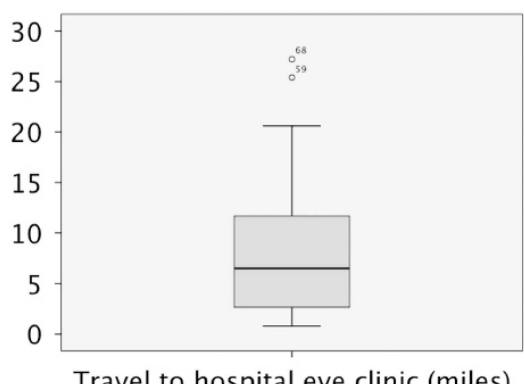

Travel to hospital eye clinic (miles)

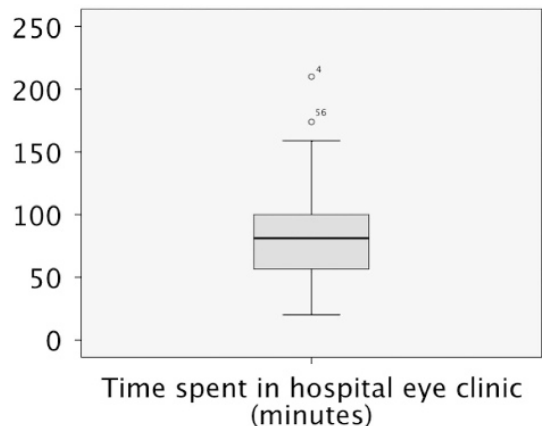

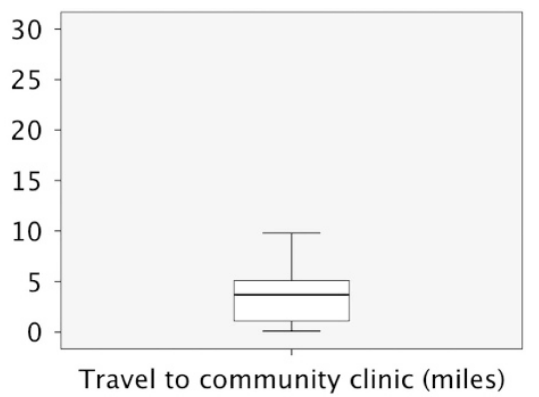

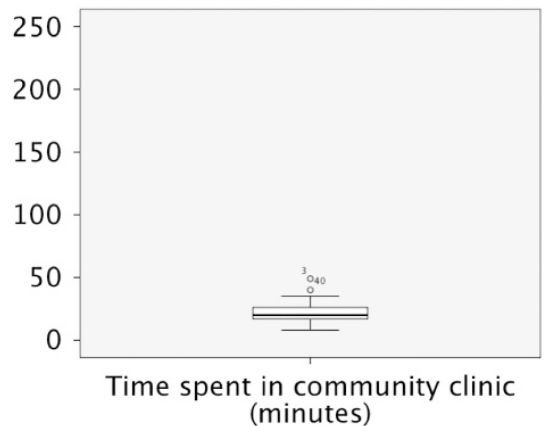

Figure 1 Top: The median distance families travelled to the hospital eye clinic was 6.4 miles, with an interquartile range (IQR) of 2.5 - 12.4 miles, whereas median travel to a local community clinic was 3.7 miles, with less variability reflected in a narrower IQR of $1.1-5.1$ miles. Bottom: The median time families spent in a hospital eye clinic was $82 \mathrm{~min}$ (IQR 55-107 min), and that in a community eye clinic $20 \mathrm{~min}$ (IQR $17-26 \mathrm{~min}$ ). 
Table 1 Variability in the distance travelled to the hospital eye clinic and (though to a lesser degree) to the community clinics between the two observation periods in November 2015 and April 2016

\begin{tabular}{|c|c|c|c|c|c|c|}
\hline \multirow[t]{2}{*}{ Clinic } & \multicolumn{2}{|c|}{ Nov-15 } & \multicolumn{2}{|c|}{ Apr-16 } & \multicolumn{2}{|c|}{ Combined data } \\
\hline & $\begin{array}{l}\text { Hospital eye } \\
\text { clinic }\end{array}$ & $\begin{array}{l}\text { Community eye } \\
\text { clinic }\end{array}$ & $\begin{array}{l}\text { Hospital eye } \\
\text { clinic }\end{array}$ & $\begin{array}{l}\text { Community eye } \\
\text { clinic }\end{array}$ & $\begin{array}{l}\text { Hospital eye } \\
\quad \text { clinic }\end{array}$ & $\begin{array}{c}\text { Community eye } \\
\text { clinic }\end{array}$ \\
\hline$n$ & 42 & 35 & 50 & 36 & 92 & 71 \\
\hline Travel distance (miles): median & 4.9 & 4.1 & 9 & 3.2 & 6.4 & 3.7 \\
\hline Interquartile range & $2.3-10.1$ & $0.8-5.1$ & $2.9-13.8$ & $1.1-5.2$ & $2.5-12.4$ & $1.1-5.1$ \\
\hline Time in clinic (min): median & 84 & 20 & 77 & 20 & 82 & 20 \\
\hline Interquartile range & $65.5-102$ & $18-25$ & $44-113$ & $16-28$ & $55-107$ & $17-26$ \\
\hline
\end{tabular}

Time spent in clinics was fairly constant.

(IQR 17 - 26) for CCs. The tariff to commissioners for HES follow-up appointments is $£ 100$, and that for CC visits is $£ 55$. Local protocols can set up a safe, family-friendly, effective and cost-efficient amblyopia pathway. Not all HES visits can be transferred to CC, but their number can be reduced. Clinical audits monitor quality of services. Further evaluations should include a larger number of sites and families to explore economic, ecological and societal impact further, for example by calculating the carbon footprint by including building energy use, travel data of staff, and patients and procurement activity data.

\section{Conflict of interest}

The authors declare no conflict of interest.

\section{Acknowledgements}

This work was supported by the NIHR Moorfields Biomedical Research Centre.

\section{Disclaimer}

The views expressed are those of the authors and not necessarily those of the NHS, the NIHR or the Department of Health. This work has not been published before. It has been accepted as poster presentation at the Annual Congress of the Royal College of Ophthalmologists, Birmingham 2016.

\section{References}

1 Malik AN, Cassels-Brown A, Wormald R, Gray JA. Better value eye care for the 21st century: the population approach. Br J Ophthalmol 2013; 97(5): 553-557.

2 Connor A, Mortimer F. The green nephrology survey of sustainability in renal units in England, Scotland and Wales. J Ren Care 2010; 36(3): 153-160.

3 Somner JE, Connor A, Benjamin L. Eyes, economics and the environment: should green issues drive changes in ophthalmic care?-yes. Eye (Lond) 2010; 24(8):

1309-1311.

R Thomas', L Brocklesby², A Coleman², V Cuchhadia², D Dunham², S Glaze², P Khandelwal ${ }^{2}$ and A Dahlmann-Noor ${ }^{1,3}$

${ }^{1}$ Moorfields Eye Hospital at Bedford Hospital, Bedford, UK

${ }^{2}$ South Essex Partnership University NHS Foundation Trust, Orthoptics Bedford, Enhanced Services Centre, Bedford Health Village, Bedford, UK

${ }^{3}$ NIHR Biomedical Research Centre at Moorfields Eye Hospital NHS Trust and UCL Institute of Ophthalmology, London, UK

Correspondence: A Dahlmann-Noor, NIHR Biomedical Research Centre at Moorfields Eye Hospital NHS Trust and UCL Institute of Ophthalmology, London, UK

Tel: +44 (0)20 7566 2013;

Fax: +44 (0)207566 2019 .

E-mail: annegret.dahlmann-noor@moorfields.nhs.uk 